\title{
Corrigendum: RecG and UvsW catalyse robust DNA rewinding critical for stalled DNA replication fork rescue
}

Maria Manosas, Senthil K. Perumal, Piero R. Bianco, Felix Ritort, Stephen J. Benkovic \& Vincent Croquette

Nature Communications 4:2368 doi: 10.1038/ncomms3368 (2013); Published 9 Sep 2013; Updated 22 Sep 2014

In the original version of this Article, the middle initial of the author Piero R. Bianco was omitted from the author information. This has now been corrected in both the PDF and HTML versions of the Article. 